\title{
DSpace@MIT
}

MIT Open Access Articles

\section{SIMULATIONS OF THE MAGELLANIC STREAM IN A FIRST INFALL SCENARIO}

The MIT Faculty has made this article openly available. Please share how this access benefits you. Your story matters.

Citation: Besla, G., N. Kallivayalil, L. Hernquist, R. P. van der Marel, T. J. Cox, and D. Keres. "SIMULATIONS OF THE MAGELLANIC STREAM IN A FIRST INFALL SCENARIO." The Astrophysical Journal 721, no. 2 (September 7, 2010): L97-L101. (C) 2010 American Astronomical Society.

As Published: http://dx.doi.org/10.1088/2041-8205/721/2/l97

Publisher: Institute of Physics/American Astronomical Society

Persistent URL: http://hdl.handle.net/1721.1/95878

Version: Final published version: final published article, as it appeared in a journal, conference proceedings, or other formally published context

Terms of Use: Article is made available in accordance with the publisher's policy and may be subject to US copyright law. Please refer to the publisher's site for terms of use. 


\title{
SIMULATIONS OF THE MAGELLANIC STREAM IN A FIRST INFALL SCENARIO
}

\author{
G. Besla $^{1}$, N. Kallivayalil ${ }^{2}$, L. Hernquist ${ }^{1}$, R. P. van der Marel ${ }^{3}$, T. J. Cox ${ }^{4}$, And D. Kereš ${ }^{1}$ \\ ${ }^{1}$ Harvard-Smithsonian Center for Astrophysics, 60 Garden Street, Cambridge, MA 02138, USA; gbesla@cfa.harvard.edu \\ ${ }^{2}$ MIT Kavli Institute for Astrophysics \& Space Research, 70 Vassar Street, Cambridge, MA 02139, USA \\ ${ }^{3}$ Space Telescope Science Institute, 3700 San Martin Drive, Baltimore, MD 21218, USA \\ ${ }^{4}$ Carnegie Observatories, 813 Santa Barbara Street, Pasadena, CA 91101, USA \\ Received 2010 July 17; accepted 2010 August 23; published 2010 September 7
}

\begin{abstract}
Recent high-precision proper motions from the Hubble Space Telescope suggest that the Large and Small Magellanic Clouds (LMC and SMC, respectively) are either on their first passage or on an eccentric long period ( $>6 \mathrm{Gyr}$ ) orbit about the Milky Way (MW). This differs markedly from the canonical picture in which the Clouds travel on a quasi-periodic orbit about the MW (period of $\sim 2 \mathrm{Gyr}$ ). Without a short-period orbit about the MW, the origin of the Magellanic Stream, a young (1-2 Gyr old) coherent stream of H I gas that trails the Clouds $\sim 150^{\circ}$ across the sky, can no longer be attributed to stripping by MW tides and/or ram pressure stripping by MW halo gas. We propose an alternative formation mechanism in which material is removed by LMC tides acting on the SMC before the system is accreted by the MW. We demonstrate the feasibility and generality of this scenario using an $\mathrm{N}$-body/smoothed particle hydrodynamics simulation with cosmologically motivated initial conditions constrained by the observations. Under these conditions, we demonstrate that it is possible to explain the origin of the Magellanic Stream in a first infall scenario. This picture is generically applicable to any gas-rich dwarf galaxy pair infalling toward a massive host or interacting in isolation.
\end{abstract}

Key words: galaxies: halos - galaxies: individual (Magellanic Clouds) - galaxies: kinematics and dynamics

Online-only material: color figures

\section{INTRODUCTION}

The Magellanic Clouds are the closest near-equal-mass interacting pair of galaxies. Optical and infrared surveys of the system present the Clouds as two distinct objects separated in space by a projected distance of $\sim 20 \mathrm{kpc}$. The $\mathrm{H}$ I distribution, however, paints a different picture. The Clouds are connected by a low-metallicity bridge of gas, referred to as the Magellanic Bridge, and share a common gaseous envelope (Putman et al. 2003; Brüns et al. 2005). The existence of such features suggests that the Clouds are a binary interacting pair. The Bridge in particular indicates that they have had a close encounter in the recent past. Toomre \& Toomre (1972) demonstrate that two isolated galaxies are capable of removing substantial amounts of material via tides, forming pronounced features such as bridges and tails. Interestingly, the system also possesses a substantial trailing Hi component, known as the Magellanic Stream (Mathewson et al. 1974). The Stream is a filamentary feature of H I gas (no stars; Guhathakurta \& Reitzel 1998) that trails behind the Clouds for at least $150^{\circ}$ across the sky (Braun \& Thilker 2004; Nidever et al. 2010). The Stream has historically been explained as the product of a tidal and/or hydrodynamic interaction between the Clouds and the Milky Way (MW; e.g., Gardiner \& Noguchi 1996; Connors et al. 2006; Mastropietro et al. 2005). This picture stems from the belief that the Clouds have traveled in an orbit that afforded multiple close passages between the Clouds and our Galaxy. However, recent Hubble Space Telescope (HST) proper motion (PM) measurements of the Clouds by Kallivayalil et al. (2006a, 2006b; hereafter K2 and K1), independently confirmed by Piatek et al. (2008), have challenged this picture. These studies suggest instead that the Clouds have, at best, completed one orbit about the MW or may even be on their first passage (Besla et al. 2007, hereafter B07). This calls for a revised interpretation of the origin of the Stream.
$\mathrm{K} 1$ determined a velocity of $378 \pm 18 \mathrm{~km} \mathrm{~s}^{-1}$ for the Large Magellanic Cloud (LMC), which is $80 \mathrm{~km} \mathrm{~s}^{-1}$ higher than that derived from theoretical models of the Stream (Gardiner \& Noguchi 1996, hereafter GN96). B07 showed that a backward integration scheme (e.g., Murai \& Fujimoto 1980) using the new velocities and an isothermal sphere model for the MW yields an orbit for the LMC with an apocenter $>200 \mathrm{kpc}$. It is, however, unlikely that the rotation curve of the MW remains flat out to such distances (e.g., Xue et al. 2008). If instead a more cosmologically motivated profile is employed (e.g., Navarro-Frenk-White (NFW) or Hernquist; Navarro et al. 1996; Hernquist 1990a) then orbital solutions with multiple pericentric passages are ruled out. In particular, if an NFW model is adopted with a mass of $10^{12} M_{\odot}$, B07 conclude that the Clouds have just experienced their first close passage past the MW.

Given the uncertainties in the adopted MW model, it is also possible that the Clouds have completed at most one orbit about our Galaxy within a Hubble time. This occurs if either the MW's mass were higher $\left(\sim 2 \times 10^{12} M_{\odot} ; \mathrm{B} 07\right)$, or if the velocity inferred from the PM measurements were substantially lower, e.g., if the velocity at the solar circle were higher (Shattow \& Loeb 2009; Reid et al. 2009). In this case, the only previous pericentric passage about the Galaxy would be $\sim 6 \mathrm{Gyr}$ ago and the apocenter of the orbit would be $\sim 400 \mathrm{kpc}$ (i.e., larger than the virial radius of the MW). These values are lower limits since these studies assume that the MW's mass is constant over time, whereas in the current LCDM paradigm our Galaxy is believed to have been half as massive $\sim 8 \mathrm{Gyr}$ ago (Wechsler et al. 2002). Depending on the mass evolution of the MW it may be impossible for the Clouds to have completed multiple passages.

Independent of which of the two orbital scenarios outlined above is correct, there is no orbital solution that brings the 
Clouds near the MW over the past 3 Gyr. However, there is strong evidence that the Stream is a young feature (1-2 Gyr). Estimates of the survivability of high velocity clouds by Heitsch \& Putman (2009) and Kereš \& Hernquist (2009) make it improbable for the Stream to have survived much longer. The Stream also exhibits surprisingly high $\mathrm{H} \alpha$ emission $(\sim 750 \mathrm{mR}$; Weiner \& Williams 1996), which implies that cloudlets within the Stream are being ablated away on a 100-200 Myr timescale and must be continuously replenished (Bland-Hawthorn et al. 2007). The lifetime of the Stream poses a problem for all past numerical models, which invoke some combination of MW tides and/or ram pressure stripping to form the Stream. These models require at least one complete orbit about the MW, implying an incompatible age of at least $6 \mathrm{Gyr}$.

As such, regardless of whether the Clouds are on their first or second passage about the MW, in the context of the origin of the Stream we are left with the same problem: how can the Stream have formed without a complete orbit about the MW? Or, more generically, how can a pronounced tail be formed from a pair of dwarfs on their first infall toward a massive host?

Based on the above considerations, we explore the following scenario using simulations. We assume that the Clouds were a stable binary system and adopt the simplifying hypothesis that the Clouds are on their first passage about the MW in order to illustrate how the Stream can form without relying on a close encounter with the MW. We postulate that the Magellanic Stream and Bridge are in fact a classical bridge and tail caused by the tidal interaction between the Clouds before they have been accreted by the MW. The MW potential shapes the orbit of the Clouds and thereby controls the appearance of the tail, causing the line-of-sight velocities and spatial location of the tail to be as observed in the Stream today.

Given these assumptions, we attempt to explain the following observed features of the Stream: (1) the absence of stars, (2) the $\sim 150^{\circ}$ extent, (3) the spatial location projected on the plane of the sky, (4) the line-of-sight velocities along its length, (5) the H I column densities, and (6) the pronounced asymmetry between the trailing and leading components.

\section{METHODOLOGY}

Simulations were carried out using the $N$-body/smoothed particle hydrodynamics code GADGET2 (Springel 2005). Star formation is not included for simplicity, but the gas is allowed to cool radiatively.

We model the MW as a static NFW potential of mass of $1.5 \times 10^{12} M_{\odot}, R_{\mathrm{vir}}=240 \mathrm{kpc}$, and a concentration parameter of 12. Dynamical friction from the MW halo is not explicitly accounted for, but is expected to have little impact on the orbit in a first passage (see B07, Figure 4). The LMC/SMC are both modeled using Hernquist profiles for their dark matter content, and exponential gaseous and stellar disks, where the scale length of the gas disk is six (LMC) or five (Small Magellanic Cloud, SMC) times that of the stellar disk. Such extended gaseous disks are not atypical for isolated dwarf galaxies (Swaters et al. 2002).

The stellar and gas masses for the Clouds are well constrained within their respective observable limits. However, the total dark matter content of these galaxies is unknown. All previous models of the Magellanic system have assumed that the LMC is tidally truncated to a radius of $15 \mathrm{kpc}$ (van der Marel et al. $2002)$, resulting in a total mass estimate of $(2-3) \times 10^{10} M_{\odot}$ (e.g., GN96; Murai \& Fujimoto 1980; Bekki \& Chiba 2005). On an orbit where MW tides are largely inconsequential, the LMC/SMC will not be truncated. Instead, we use current halo
Table 1

LMC/SMC Initial Conditions Adopted

\begin{tabular}{lcc}
\hline \multicolumn{1}{c}{ Property } & LMC & SMC \\
\hline$M_{*}\left(M_{\odot}\right)$ & $2.2 \times 10^{9}$ & $1.3 \times 10^{8}$ \\
$M_{\text {gas }}\left(M_{\odot}\right)$ & $1.4 \times 10^{9}$ & $1.1 \times 10^{9}$ \\
$M_{\text {halo }}\left(M_{\odot}\right)^{\mathrm{a}}$ & $1.8 \times 10^{11} M_{\odot}$ & $2.5 \times 10^{10}$ \\
$V 200\left(\mathrm{~km} \mathrm{~s}^{-1}\right)$ & 82 & 42 \\
Concentration & 9 & 15 \\
Stellar scale length $(\mathrm{kpc})$ & 1.7 & 0.7 \\
Gas scale length $(\mathrm{kpc})$ & 10.2 & 3.6 \\
$N_{\text {stars }}$ & 100000 & 100000 \\
$N_{\text {gas }}$ & 300000 & 300000 \\
$N_{\text {halo }}$ & 100000 & 100000 \\
\hline
\end{tabular}

Note.

a The total halo mass is determined using the observed stellar mass of the LMC (SMC) $M_{*}=3 \times 10^{9} M_{\odot}\left(3 \times 10^{8} M_{\odot}\right)$ (van der Marel et al. 2002; Stanimirović et al. 2004) and the relations from Wang et al. (2006). Note that the observed $M_{*}$ is less than that quoted in this table in order to account for star formation in future studies.

occupation models to relate the observed stellar mass of the LMC to its original halo mass before infall into the MW halo (van den Bosch et al. 2003; Conroy et al. 2006; Wang et al. 2006). In Table 1, we summarize our adopted model properties. The LMC/SMC are found to have infall masses an order of magnitude larger than employed in previous models.

\section{SIMULATION RESULTS AND COMPARISONS WITH DATA}

We believe that the key to understanding the origin of the Stream lies in understanding the interaction history of the Clouds themselves. The majority of previous models of the Stream have assumed that the SMC is in a circular orbit about the LMC (separation of $\sim 20 \mathrm{kpc}$ ). Using the cosmologically expected infall masses, the dynamical friction timescale for such an orbit would be much less than a Hubble time, so the SMC's orbit about the LMC cannot be circular. The idea that collisions between the Clouds have caused material to be loosely bound to the Clouds is not novel (GN96; Heller \& Rohlfs 1994). However, the morphology resulting from collisions between the Clouds as the SMC travels in a highly eccentric orbit about the LMC, independent of the MW, has never been explored.

We have used the current projected separation between the Clouds $(23 \mathrm{kpc})$, and the observed relative velocity $(\sim 105 \pm$ $42 \mathrm{~km} \mathrm{~s}^{-1} ; \mathrm{K} 2$ ) to constrain the orbital history of the SMC about the LMC. The resulting orbit is nearly parabolic $(e=0.7)$, with an apocenter of $\sim 100 \mathrm{kpc}$ (see Figure 1). The SMC disk is oriented $90^{\circ}$ with respect to its orbital plane about the LMC. If the SMC were in a coplanar, retrograde orbit about the LMC, no material would be removed with this orbital configuration. This implies that dispersion supported material (e.g., dark matter or stellar halo) will be unaffected within the SMC's disk radius.

The presented orbital solution is not unique; however, a highly eccentric orbit is required to prevent the Clouds from merging. Moreover, these orbits are cosmologically typical (see, e.g., Benson 2005; Wetzel 2010). Any eccentric orbit that allows for high speed encounters between the Clouds will yield similar bridge and tail structures.

We simulated the interaction between the Clouds as the SMC travels along the orbit shown in Figure 1 (top left) starting > 6 Gyr ago. At each close passage between the Clouds, gas is 

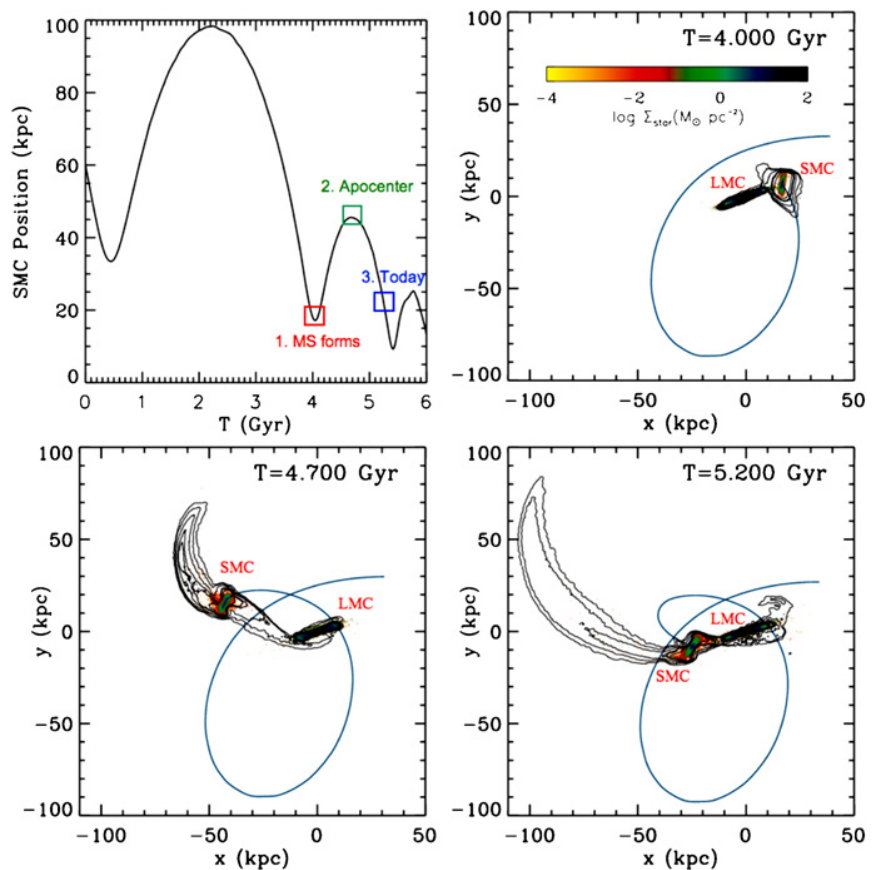

Figure 1. Orbit of the SMC about the LMC (without the MW). Top left: the separation between the Clouds as a function of time. The chosen orbit is highly eccentric $(e=0.7)$ and decays rapidly owing to dynamical friction. The Stream forms at $4 \mathrm{Gyr}$ (red box) and the time today would correspond to $\sim 5.2 \mathrm{Gyr}$ (blue box). Subsequent panels: the H I gas column density is plotted as contours over the stellar distribution for specific points in the orbit. Gas contours span a range of $10^{18}-10^{20} \mathrm{~cm}^{-2}$, where each contour represents an increase in column density by a factor of 1.5 . H I gas is identified as gas at temperatures below $12,000 \mathrm{~K}$ and column densities larger than $10^{18} \mathrm{~cm}^{-2}$, although a background ionizing field is not included. A bridge of gas connecting the Clouds and a $100 \mathrm{kpc}$ long gaseous stream with no stellar counterpart is formed without the aid of MW tides or ram pressure.

(A color version of this figure is available in the online journal.)

removed from the SMC by LMC tides, forming a tidal tail and bridge. However, as the Clouds move apart, the tidal material falls back toward the LMC/SMC disks. These features are thus transient phenomena. Little material is removed from the LMC, despite its extended gaseous disk component: SMC tides are ineffective.

The pericentric passage at $4 \mathrm{Gyr}$ in Figure 1 (top left) results in significant gas removal from the outer regions of the SMC's extended gas disk, whereas the smaller stellar disk remains intact. As the SMC travels away from the LMC, material that was tidally removed by the LMC stretches out to distances as large as $100 \mathrm{kpc}$ from the Clouds. This material will eventually form the Stream (Figure 1, bottom right). Hence, an extended gaseous tail is produced without the aid of MW tides or ram pressure.

We assume that the Clouds have been an interacting pair for a significant fraction of a Hubble time and have crossed the virial radius $(240 \mathrm{kpc}$ ) of our Galaxy $\sim 1 \mathrm{Gyr}$ ago. From Figure 1, a $100 \mathrm{kpc}$ long tail is formed after $\sim 5 \mathrm{Gyr}$. We therefore stop the simulation $1 \mathrm{Gyr}$ earlier (i.e., at the time step corresponding to the bottom left panel of Figure 1) and place the binary system outside the virial radius of the MW. We then allow the Clouds to travel to their current observed locations on an orbit consistent within $1 \sigma$ of the PMs of the LMC (K1; Piatek et al. 2008). We did not attempt to reproduce the SMC PM determined by K2 and Piatek et al. (2008) since they are discrepant. However, the resulting SMC line-of-sight velocity agrees well with the observed value.
In Figure 2, we show the resulting stellar distribution (top) and $\mathrm{H}$ I gas column density map (middle) for our simulated stream: there are no observable stars in the simulated stream and the stream extends $\sim 150^{\circ}$. The white line indicates the current location of the observed Stream and is well matched by the simulation. Note that the past orbits (yellow lines) are not co-located with the simulated stream, as expected since the north component of the LMC PM vector is not aligned with the Stream (see B07, Figure 9). This spatial mismatch is a natural result of our model: material is removed from the SMC along the LMC-SMC binary orbital plane, which is not coplanar with the orbital plane of the Clouds about the MW. The SMC disk was oriented $90^{\circ}$ to the LMC/SMC binary plane in order to maximize this offset. This further implies that the SMC is seen edge on from our viewing perspective, explaining its surprisingly large observed line-of-sight depth ( $\sim 5 \mathrm{kpc}$; Subramanian \& Subramanian 2009).

Figure 2 (bottom) illustrates the resulting line-of-sight velocities for the simulated stream. The white line is a fit to the data (Putman et al. 2003; Nidever et al. 2008, hereafter N08) and shows good agreement with the simulation. The line-of-sight velocities along the new orbits (yellow lines) are much larger than those observed along the Stream: since the Stream and the orbits are not co-located, their line-of-sight velocities are not similar (see also B07, Figure 20). The MW's gravitational field serves to stretch the stream and modify its galactocentric distance: the tip of the simulated stream is located $\sim 100-140 \mathrm{kpc}$ away. Using these distance estimates, we find that the mass in our simulated stream matches the observed value $\left(4.8 \times 10^{8} M_{\odot}\right.$; Brüns et al. 2005) to within a factor of two.

The simulated gas column densities range from $10^{18}$ $10^{21} \mathrm{~cm}^{-2}$ as observed, although the exact column density gradient along the length of the Stream is not reproduced. The column density is not homogeneous across the width of the simulated stream: the inclusion of metal cooling, ionization, and confinement/interaction by/with the ambient MW halo gas will likely aid in reproducing the bifurcated, filamentary nature of the observed Stream.

The leading component of the simulated stream is much smaller than the trailing component, as observed. This arises because the leading tidal arm from the SMC falls toward the LMC, while the tail continues to grow (Figure 1). However, the leading component in the model is not in the correct location on the sky when compared to observations. This material leads the orbit, which is not aligned with the Stream. Consequently, this apparent problem will also occur in the traditional tidal models of, e.g., Rǔžička et al. (2009), Bekki (2008), Connors et al. (2006), Bekki \& Chiba (2005), and Gardiner \& Noguchi (1996). This indicates that hydrodynamic processes such as ram pressure (Mastropietro et al. 2005) are needed to shape the final appearance of the simulated stream.

\section{DISCUSSION AND CONCLUSIONS}

We have shown that tidal interactions between the Clouds are sufficient to remove a substantial amount of material from the SMC without the aid of MW tides or hydrodynamic interactions. Our goal was not to reproduce every detail of the Stream, but merely to show that a plausible Stream model can be formed without a previous passage about the MW. Nevertheless, many features of the simulation fit the data remarkably well.

We have explained the absence of an observable (surface density larger than $0.5 M_{\odot} \mathrm{pc}^{-2}$ ) stellar counterpart to the Stream by placing the SMC on an orbit about the LMC with a 


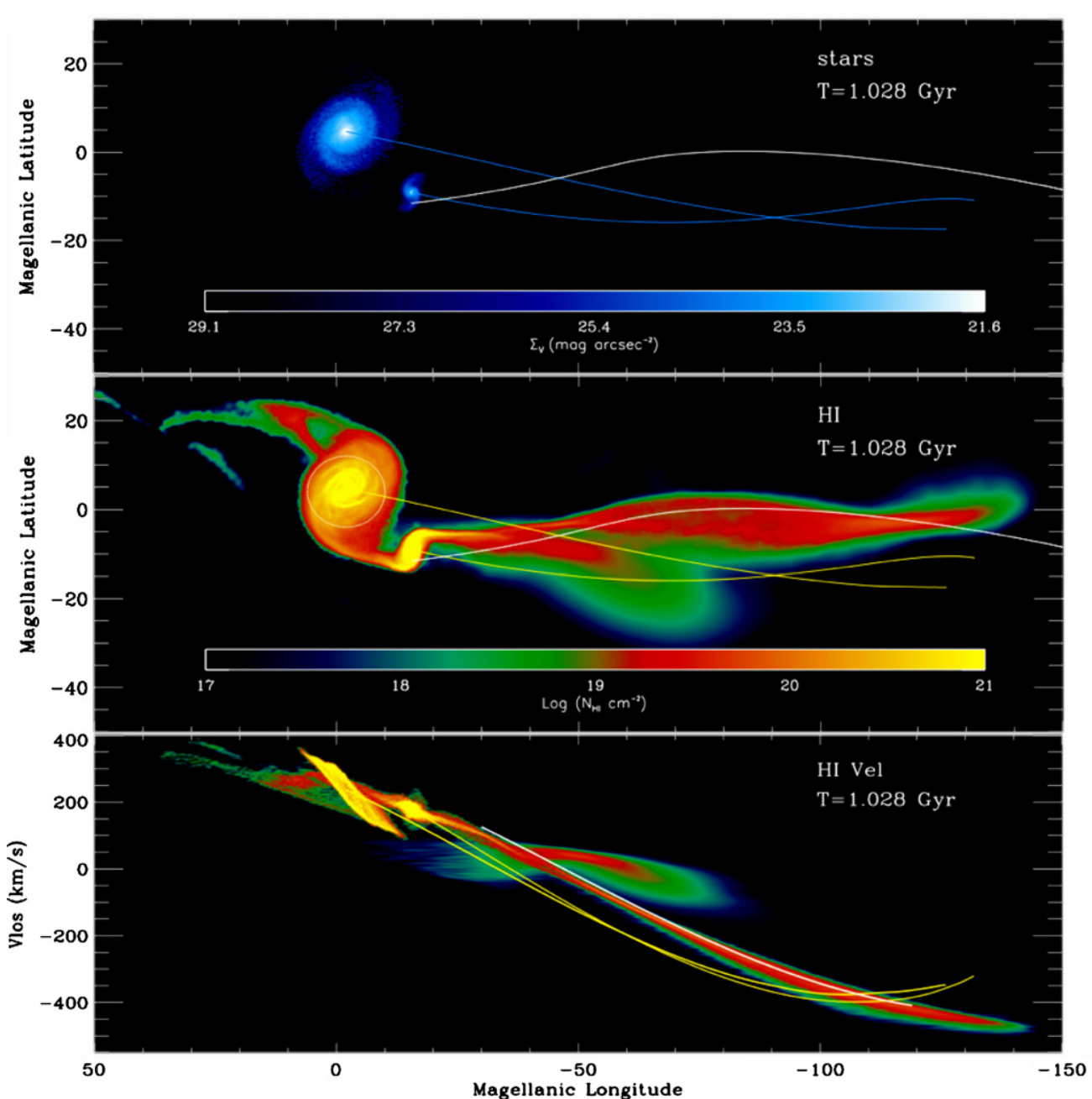

Figure 2. Stellar surface brightness, H I gas column densities, and line-of-sight velocities of the simulated Magellanic system. Top panel: the resulting stellar distribution is projected in Magellanic coordinates (N08), a variation of the Galactic coordinate system where the Stream is straight. The distribution is color-coded in terms of $V$-band surface brightness. The past orbit of the LMC/SMC is indicated by the blue lines. Middle panel: the HI gas column densities of the simulated stream range from $10^{18}$ to $10^{21} \mathrm{~cm}^{-2}$, as expected (Putman et al. 2003). The white circle indicates the observed extent of the LMC's H I disk: the simulated LMC is more extended than observed, indicating ram pressure likely plays a role to truncate the disk. In both the top and middle panels, the solid white line indicates the past orbit of the SMC according to the old theoretically derived PMs (GN96) which was a priori chosen to trace the Stream on the plane of the sky. The true orbits (determined by all PM measurements) for the LMC/SMC are indicated by the yellow lines. Bottom panel: the line-of-sight velocities along the simulated stream are plotted and color-coded based on H I column density, as in the middle panel. The white line is a fit to the observed data (N08). The LMC disk is too extended, causing a larger velocity spread than observed. The line-of-sight velocities along the past orbits of the LMC/SMC are indicated by the yellow lines, which do not follow the true velocities along the Stream (e.g., B07, Figure 20). The Stream is kinematically distinct from the orbits of the Clouds

(A color version of this figure is available in the online journal.)

large impact parameter, which allows its compact stellar disk to remain intact (see also, Yoshizawa \& Noguchi 2003). The $150^{\circ}$ extent of the Stream is reproduced owing to the eccentric orbit of the SMC about the LMC (apocenter $\sim 100-50 \mathrm{kpc}$ ) and MW tides after the system has been accreted. Such an eccentric orbit is likely only viable in a first infall scenario, as MW tides would have disrupted the system at the previous pericentric approach. The spatial location of and velocity gradient along the simulated stream are correct and offset from those of the orbits: material is removed in the LMC/SMC binary plane, which is not coplanar with the orbit of the Clouds about the MW. The simulated $\mathrm{HI}$ column densities also match the observations, although the exact column density gradient is not reproduced without additional physics. Finally, the strong asymmetry between the leading and trailing stream components is a natural consequence of a classical tidal bridge and tail scenario.

There are, however, other features that require additional physics to address, such as the claim of correlated bursts of star formation in both Clouds (Harris \& Zaritsky 2009). In our new scenario, correlated bursts of star formation may correspond to close passages between the Clouds rather than with the MW. Furthermore, without ram pressure and stellar feedback we cannot explore the claim by N08 that half of the Stream originates from the LMC as a stellar outflow (see also, B07 and Olano 2004). We intend to examine these issues and more detailed comparisons of our simulations to the Magellanic system in future studies. However, we note that recent metallicity measurements at the tip of the Stream (Fox et al. 2010) indicate that the Stream is extremely metal poor and thus inconsistent with a stellar outflow scenario. Fox et al. (2010) also find that the oxygen abundance at the tip is more consistent with that of the SMC rather than the LMC. As such, a model where the Stream originates primarily from the SMC is not ruled out by observations of the Stream.

The simulation results presented here with respect to the LMC/SMC interaction have broader applicability than just to 
the Magellanic system. Within the current LCDM paradigm halos at all scales are expected to build up their mass hierarchically. Interacting dwarf galaxies are therefore cosmologically expected both in isolation and within larger halos (Simha et al. 2010; D’Onghia et al. 2009; Knebe et al. 2006). The isolated interacting Magellanic-type galaxies NGC 4490/85, which are surrounded by an extended H i envelope (Clemens et al. 1998), and the interacting M51/NGC 5195 pair (Hernquist 1990b) may be observational analogs of our initial LMC/SMC system. The presented model thus illustrates that dwarf-dwarf galaxy tidal interactions are a powerful mechanism to morphologically change dwarf galaxies without the need for repeated interactions with a massive host.

\section{REFERENCES}

Bekki, K. 2008, ApJ, 684, L87

Bekki, K., \& Chiba, M. 2005, MNRAS, 356, 680

Benson, A. J. 2005, MNRAS, 358, 551

Besla, G., Kallivayalil, N., Hernquist, L., Robertson, B., Cox, T. J., van der Marel, R. P., \& Alcock, C. 2007, ApJ, 668, 949 (B07)

Bland-Hawthorn, J., Sutherland, R., Agertz, O., \& Moore, B. 2007, ApJ, 670, L109

Braun, R., \& Thilker, D. A. 2004, A\&A, 417, 421

Brüns, C., et al. 2005, A\&A, 432, 45

Clemens, M. S., Alexander, P., \& Green, D. A. 1998, MNRAS, 297, 1015

Connors, T. W., Kawata, D., \& Gibson, B. K. 2006, MNRAS, 371, 108

Conroy, C., Wechsler, R. H., \& Kravtsov, A. V. 2006, ApJ, 647, 201

D’Onghia, E., Besla, G., Cox, T. J., \& Hernquist, L. 2009, Nature, 460, 605

Fox, A. J., Wakker, B. P., Smoker, J. V., Richter, P., Savage, B. D., \& Sembach, K. R. 2010, ApJ, 718, 1046

Gardiner, L. T., \& Noguchi, M. 1996, MNRAS, 278, 191 (GN96)

Guhathakurta, P., \& Reitzel, D. B. 1998, in ASP Conf. Ser. 136, Galalctic Halos: A UC Santa Cruz Workshop, ed. D. Zaritsky (San Francisco, CA: ASP), 22 Harris, J., \& Zaritsky, D. 2009, AJ, 138, 1243

Heitsch, F., \& Putman, M. E. 2009, ApJ, 698, 1485

Heller, P., \& Rohlfs, K. 1994, A\&A, 291, 743
Hernquist, L. 1990a, ApJ, 356, 359

Hernquist, L. 1990b, in Dynamics and Interactions of Galaxies, ed. R. Wielen (Berlin: Springer), 108

Kallivayalil, N., van der Marel, R. P., \& Alcock, C. 2006a, ApJ, 652, 1213 (K2)

Kallivayalil, N., van der Marel, R. P., Alcock, C., Axelrod, T., Cook, K. H., Drake, A. J., \& Geha, M. 2006b, ApJ, 638, 772 (K1)

Kereš, D., \& Hernquist, L. 2009, ApJ, 700, L1

Knebe, A., Power, C., Gill, S. P. D., \& Gibson, B. K. 2006, MNRAS, 368, 741

Mastropietro, C., Moore, B., Mayer, L., Wadsley, J., \& Stadel, J. 2005, MNRAS, 363, 509

Mathewson, D. S., Cleary, M. N., \& Murray, J. D. 1974, ApJ, 190, 291

Murai, T., \& Fujimoto, M. 1980, PASJ, 32, 581

Navarro, J. F., Frenk, C. S., \& White, S. D. M. 1996, ApJ, 490, 493

Nidever, D. L., Majewski, S. R., \& Burton, W. B. 2008, ApJ, 679, 432 (N08)

Nidever, D. L., Majewski, S. R., \& Burton, W. B. 2010, (arXiv:1009.0001)

Olano, C. A. 2004, A\&A, 423, 895

Piatek, S., Pryor, C., \& Olszewski, E. W. 2008, ApJ, 135, 1024

Putman, M., Staveley-Smith, L., Freeman, K. C., Gibson, B. K., \& Barnes, D. G. 2003, ApJ, 586, 170

Reid, M. J., et al. 2009, ApJ, 700, 137

Rủžička, A., Theis, C., \& Palouš, J. 2009, ApJ, 691, 1807

Shattow, G., \& Loeb, A. 2009, MNRAS, 392, L21

Simha, V., Weinberg, D. H., Davé, R., Gnedin, O. Y., Katz, N., \& Kereš, D. 2010, MNRAS, 399, 650

Springel, V. 2005, MNRAS, 364, 1105

Stanimirović, S., Staveley-Smith, L., \& Jones, P. A. 2004, ApJ, 604, 176

Subramanian, S., \& Subramanian, A. 2009, A\&A, 496, 399

Swaters, R. A., van Albada, T. S., van der Hulst, J. M., \& Sancisi, R. 2002, A\&A, 390,829

Toomre, A., \& Toomre, J. 1972, ApJ, 178, 623

van den Bosch, F. C., Mo, H. J., \& Yang, X. 2003, MNRAS, 345, 923

van der Marel, R. P., Alves, D. R., Hardy, E., \& Suntzeff, N. B. 2002, AJ, 124, 2639

Wang, L., Li, C., Kauffmann, G., \& De Lucia, G. 2006, MNRAS, 371, 537

Wechsler, R. H., Bullock, J. S., Primack, J. R., Kravtsov, A. V., \& Dekel, A. 2002, ApJ, 568, 52

Weiner, B. J., \& Williams, T. B. 1996, AJ, 111, 1156

Wetzel, A. 2010, arXiv:1001.4792

Xue, X. X., et al. 2008, ApJ, 684, 1143

Yoshizawa, A. M., \& Noguchi, M. 2003, MNRAS, 339, 113 